СТАТЬИ

УДК 553.98:621.6

МЕТОДИКА ДИАГНОСТИКИ ФАКТИЧЕСКОГО СОСТОЯНИЯ И НЕИСПРАВНОСТЕЙ УСТАНОВОК ЭЛЕКТРОЦЕНТРОБЕЖНЫХ НАСОСОВ (УЭЦН) В РЕАЛЬНОМ ВРЕМЕНИ С ИСПОЛЬЗОВАНИЕМ ПРОМЫСЛОВЫХ, ЭЛЕКТРОТЕХНИЧЕСКИХ И ГЕОЛОГИЧЕСКИХ ПАРАМЕТРОВ

Большунов А.В., Мостакалов К.А.

Национальный исследовательский Томский политехнический университет, Томск, e-mail: artembolschunov@yandex.ru

Статья посвящена методике диагностики неисправностей установок электроцентробежных насосов с использованием электротехнических и геологических параметров. Электрический погружной насос является важным цифровым механическим оборудованием на нефтяных месторождениях, что рождает необходимость проведения мониторинга состояния и диагностики неисправностей системы работы электроцентробежных насосов. Автор предлагает онлайн-методику, которая основывается на электрических параметрах и параметрах производства. Чтобы реализовать диагностику неисправностей электроцентробежных насосов в земле, были разработаны алгоритмы с учетом особенностей извлечения. Данные алгоритмы объединяют электрические параметры с производственными параметрами, которые разделены недавно разработанным газожидкостным двухфазным расходомером. Между тем электрические параметры являются основными, а производственные параметры являются вспомогательными из них. Функции параметров извлекаются из различных неисправностей с помощью цифровой обработки сигналов, а исследования точно контролируют девять типичных рабочих условий неисправностей. Важно отметить, что предложенный метод значительно снижает воздействие на датчики в забое скважины, и данные испытаний показывают, что предложенный способ обладает такими преимуществами, как низкая стоимость и высокая эффективность. В этой статье изучаются принцип работы УЭЦН и теория алгоритма диагностики неисправностей. В исследовании предлагается методика создания базы признаков текущих электрических параметров и параметров производства в различных режимах неисправности УЭЦН. Чтобы уменьшить влияние производственной среды и комплекса забойных условий, в грунте получены все параметры, которые могут отражать состояние подземных работ. Кроме того, в исследовании разрабатываются алгоритмы, основанные на полевом опыте и схематических диаграммах, которые указывают на то, что метод эффективно улучшает добычу нефти.

Ключевые слова: установка погружного электроцентробежного насоса, электротехнические параметры, геологические параметры, неисправности УЭЦН, диагностики неисправностей УЭЦН

\title{
METHODOLOGY FOR REAL-TIME DIAGNOSTICS OF ACTUAL STATE AND FAULTS OF ELECTRIC CENTERING PUMP PLANTS USING FIELD, ELECTRICAL AND GEOLOGICAL PARAMETERS
}

Bolshunov A.V., Mostakalov K.A.

National Research Tomsk Polytechnic University,Tomsk, e-mail: artembolschunov@yandex.ru

The article is devoted to the method for diagnosing malfunctions of electric centrifugal pump installations using electrical and geological parameters. The electric submersible pump is an important digital mechanical equipment in oil fields, which gives rise to the need for monitoring the condition and diagnosing malfunctions of the operation system of electric centrifugal pumps. The author offers an online technique, which is based on electrical parameters and production parameters. These algorithms combine electrical parameters with production parameters, which are separated by a newly developed gas-liquid two-phase flow meter. Meanwhile, electrical parameters are the main ones, and production parameters are auxiliary ones. Parameter functions are extracted from various faults using digital signal processing, and research accurately controls nine typical fault operating conditions. It is important to note that the proposed method significantly reduces the impact on the sensors in the bottom of the well, and test data show that the proposed method has such advantages as low cost and high efficiency. This article explores how the ESP works and the theory of the fault diagnosis algorithm. The study proposes a methodology for creating a library of signs of current electrical parameters and production parameters in various ESP fault modes. To reduce the influence of the production environment and the complex of bottomhole conditions, all parameters that can reflect the state of underground work have been obtained in the ground. In addition, the study develops algorithms based on field experience and schematic diagrams that indicate that the method effectively improves oil production.

Keywords: submersible electric centrifugal pump installation, electrical parameters, geological parameters, ESP faults, diagnostics of ESP faults

С постоянным развитием науки и техники люди все больше внимания уделяют нехватке энергии и загрязнению окружающей среды. В частности, эксплуатация нефтяных месторождений предъявляет более высокие требования к добыче, разработке, оценке и другим технологиям. Установки электроцентробежных насосов (УЭЦН) широко используются на нефтяных месторождениях благодаря их преимуществам, 
связанным с высоким подъемом, большим рабочим объемом и удобством управления. Поэтому очень важно провести исследования по теории и технологии диагностики неисправностей УЭЦН. В настоящее время обнаружение рабочего состояния и диагностика неисправностей УЭЦН в точке источника сигнала в основном включают в себя традиционные методы обнаружения и современные методы обнаружения [1, с. 250].

Традиционные методы обнаружения включают в себя текущий метод карты и схему контроля эффективности управления системой. Современные методы обнаружения в основном включают в себя анализ вибрации и диагностику рабочего состояния. Существует много исследований методов анализа различных видов сигналов. В статье изучаются принцип работы УЭЦН и теория алгоритма диагностики неисправностей. В исследовании предлагается методика создания базы признаков текущих электрических параметров и параметров производства в различных режимах неисправности УЭЦН.

\section{Материалы и методы исследования}

\section{Системные компоненты УЭЦН}

Мощность заземления подается в погружной электродвигатель через кабель погружного насоса под трубой в скважину. Затем двигатель приводит многоступенчатый центробежный насос во вращение для создания центробежной силы, поднимая сырую нефть в скважине на землю. УЭЦН состоит из скважинной, наземной части и промежуточных частей. Наземная часть состоит из трансформаторной группы, автоматической консоли и вспомогательного оборудования, а промежуточная часть состоит из кабеля и трубки. Стальной трос используется для крепления кабеля и колонны труб. Наконец, скважинная часть - это в основном блок УЭЦН. Скважинная часть это основной блок УЭЦН [2].

\section{Шесть наборов параметров УЭЦН}

Недавно разработанный трубчатый газожидкостный двухфазный расходомер обладает преимуществом измерения добычи газа и добычи жидкости в режиме реального времени. Значение соотношения газ - жидкость можно получить, рассчитав эти два набора параметров. Однофазный ток и однофазное напряжение можно получить из электрического шкафа. Давление эксплуатационной колонны УЭЦН получа- ют из устья скважины с помощью датчика давления. Шесть параметров передаются в центр управления через терминал оборудования через интернет.

По сравнению с преимуществами получения сигнала в традиционных методах обнаружения и современных методах обнаружения выбранные шесть наборов параметров связаны с параметрами в диагностическом приборе. В этой статье приняты текущие параметры в традиционном методе обнаружения и некоторые связанные параметры в современном методе обнаружения. Кроме того, обмотка, температура, ток утечки и вибрация двигателя в современном методе обнаружения оказывают определенное влияние на рабочий ток и напряжение двигателя. Температура всасывания на входе, давление на выходе и давление на входе все еще оказывают влияние, которое отражено в производственных параметрах. Следовательно, диагностический прибор обеспечивает теоретическую основу для выбора параметров. Однако эти параметры диагностического прибора получены из скважины.

\section{Механизм измерения сигнала}

Некоторые формулы используются для определения релевантности попытки и надежности метода измерения [3, с. 315]. Давление всасывания насоса равно высоте столба жидкости над впускным отверстием плюс давление рукава.

$$
Д_{\mathrm{sc}}=\mathrm{H}+\Delta Д_{\mathrm{p}}
$$

где Д вс - давление всасывания насоса; Н - высота столба жидкости над всасывающим отверстием; $Д_{p}$ - давление в рукаве.

Таким образом, давление в забое скважины может быть выражено как:

$$
Д_{3 \mathrm{c}}=Д_{\mathrm{p}}+\left(\mathrm{H}-\mathrm{1}_{\mathrm{p}}\right) * \overline{\mathrm{p}}
$$

где Д

Н - высота насоса;

$\overline{\mathrm{p}}$ - средняя плотность жидкости.

Согласно модели прогнозирования производительности, выход жидкости под давлением на входе в насос может быть получен путем

$$
\mathrm{Q}=\mathrm{f}\left(\text { Д }_{3 \mathrm{c}}\right),
$$

где Q - выходной сигнал, который можно выразить как

$$
\mathrm{Q}=\mathrm{Q}_{1}+\mathrm{Q}_{\mathrm{g}},
$$

где $\mathrm{Q}_{1}$ - производство жидкости.

$\mathrm{Q}_{\mathrm{g}}-$ производство газа; 
$\mathrm{u}_{1}, \mathrm{u}_{2}, \ldots, \mathrm{u}_{8}, \mathrm{u}_{9}$ - это набор неисправностей, и неисправности, включая недогрузку, перегрузку, газовый эффект, блокировку газа, недостаточную подачу жидкости в нефтяных скважинах, утечку инструментальной колонны, напряжение выше номинального значения, напряжение ниже номинального значения, изменения давления всасывания, тока, напряжения, давления в забое скважины и выхода жидкости, которые очевидны. Поэтому эти шесть параметров используются для оценки условий работы УЭЦН. Эти отношения могут быть выражены как [4]:

$\mathrm{U}=\left\{\mathrm{u}_{1}, \mathrm{u}_{2}, \ldots, \mathrm{u}_{8}, \mathrm{u}_{9}\right\}=\left\{Д_{\mathrm{Bc}}, \mathrm{I}, \mathrm{V}, Д_{3 \mathrm{c}}, \mathrm{Q}\right\}$.

\section{Алгоритмы распознавания признаков}

Особенности параметров УЭЦН анализируются из разных условий для разных неисправностей. Принципиальная схема алгоритмов распознавания объектов показана на рис. 1. Кроме того, главное суждение принимает методы предварительного суждения и методы точного анализа. Метод предварительного решения может распознать, есть ли ошибка в простом суждении. Метод точного анализа может точно диагностировать модели ошибок.

Описаны несколько категорий неисправностей и извлечены тренды изменения параметров. Изменяющиеся тенденции включают в себя ожидание и дисперсию. Параметры включают электрические параметры и параметры про- изводства. Согласно теории вероятностей и статистики в текущем исследовании ожидание отражает среднее значение случайной величины. Максимальное значение и минимальное значение отражают отклонение данных. На самом деле временная область используется для измерения степени между случайной величиной и ее ожиданием.

\section{Типичные ошибки}

В этом разделе анализируются девять типичных неисправностей, встречающихся в процессе производства, которые в основном представлены электрическими параметрами и производственными параметрами. С учетом состояния поля составлены девять принципиальных схем, которые могут отражать изменяющийся тренд параметров. Похожая методика рассмотрена в [5, с. 315$]$. По сравнению с изменением фактических параметров приложения эти диаграммы могут использоваться в качестве справочных. Исходное состояние девяти проанализированных принципиальных схем является нормальным, поэтому изменения в нормальном диапазоне показаны в первом сегменте параметров.

Случай 1: В нормальном состоянии расчетная мощность и фактическая мощность почти равны нормальному значению. Кроме того, давление масла и температура на устье находятся в нормальном диапазоне колебаний, и на рис. 2 показано, что параметры находятся в нормальном диапазоне.

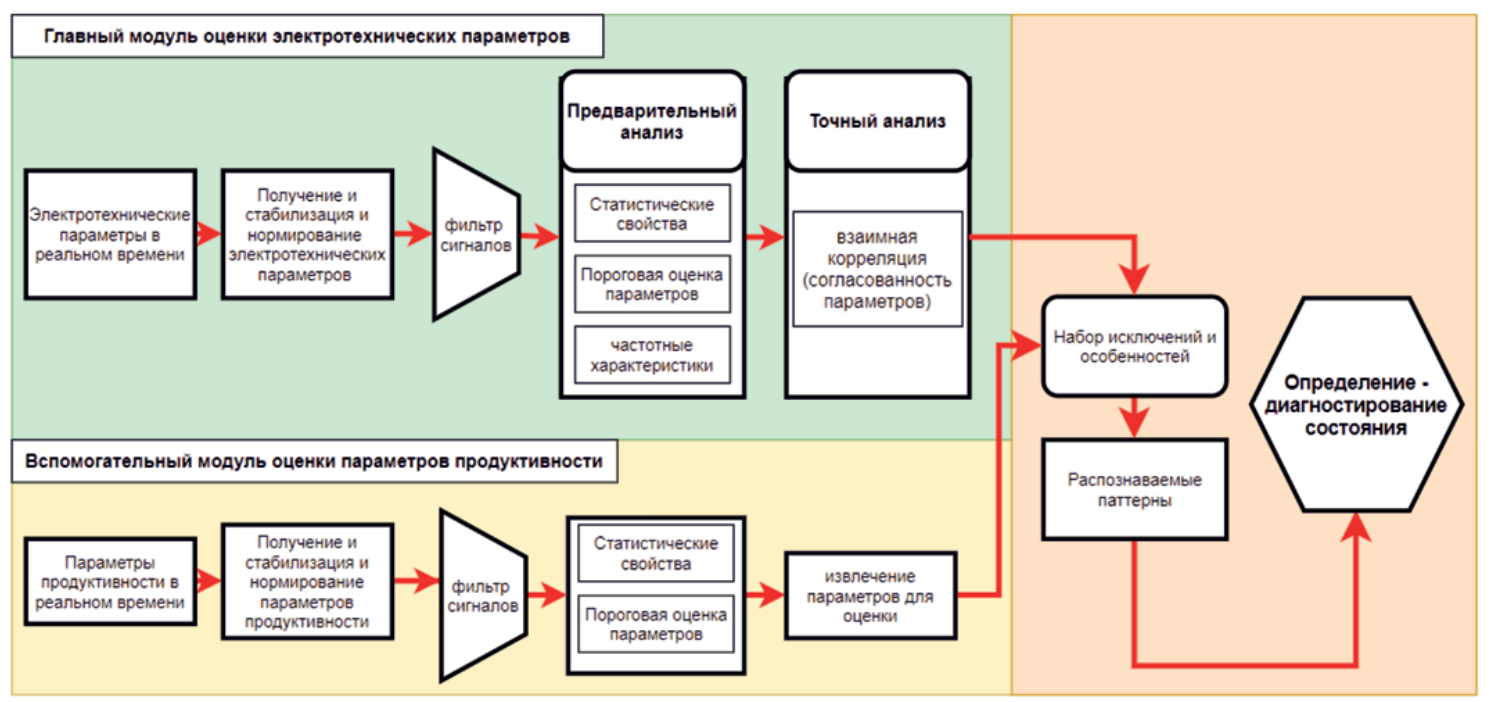

Рис. 1. Приничипиальная схема алгоритмов распознавания объектов 

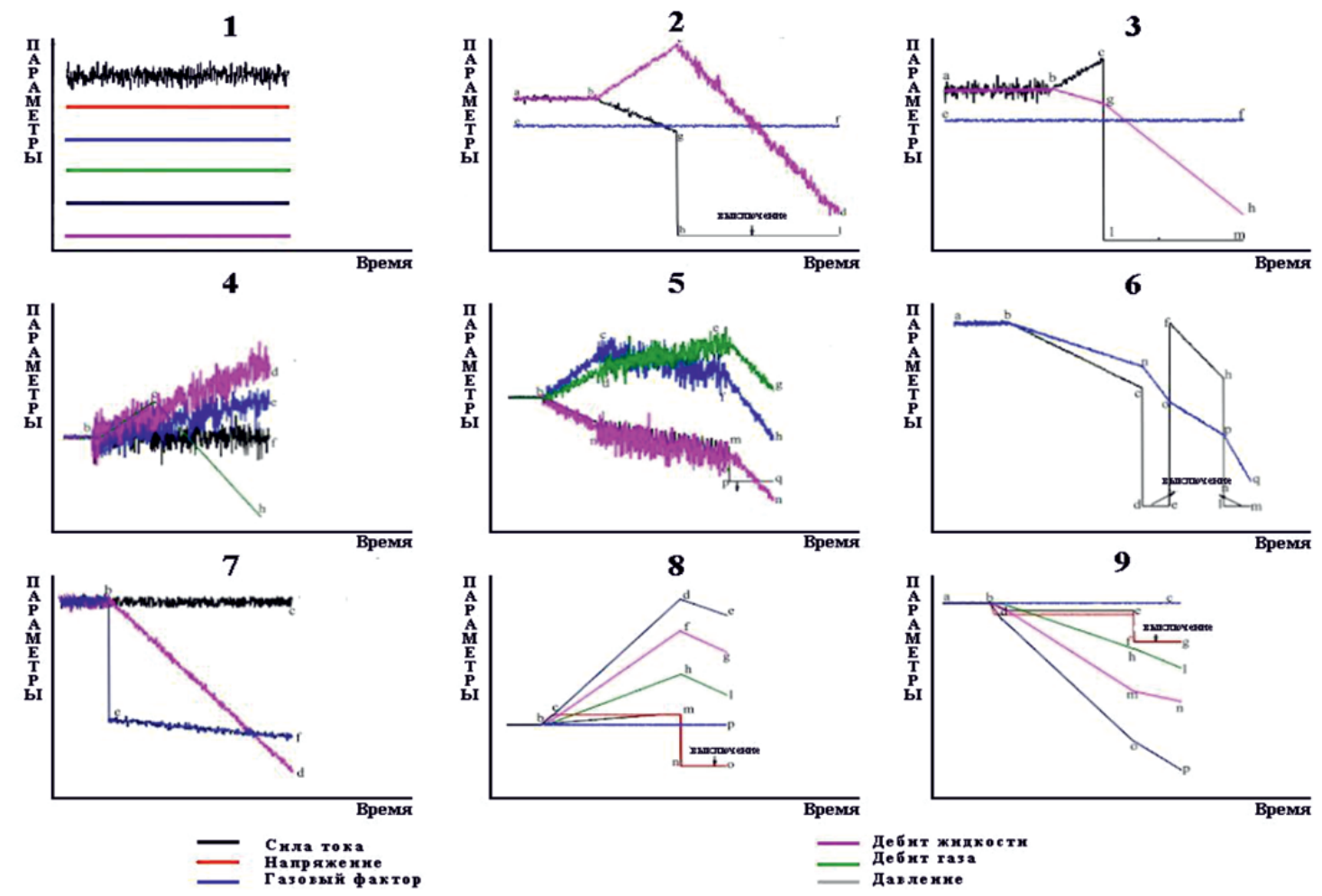

Рис. 2. Типичные неисправности, встречающиеся в процессе работы УЭЦН

Случай 2: Сегмент $\mathrm{a}-\mathrm{b}$, показанный на рис. 2, указывает на то, что двигатель работает нормально. Во время непрерывной работы однофазный ток уменьшается из-за УЭЦН в состоянии недостаточной нагрузки, как показано в сегменте b-g. Когда ток падает до заданного значения отключения при недогрузке, двигатель некоторое время продолжает работать при заданном значении недогрузки. Сегмент $\mathrm{g}-\mathrm{h}$ указывает на то, что ток неожиданно обнуляется после длительной работы в режиме недостаточной нагрузки. Производство жидкости увеличивается из-за небольшого выбора нагрузки, как показано при нормальных условиях, поэтому первый сегмент параметров находится в нормальном диапазоне изменений.

Случай 3: аналогично, сегмент $\mathrm{a}-\mathrm{b}$, показанный на рис. 2, указывает на то, что УЭЦН работает хорошо. Во время непрерывной работы ток увеличивается из-за перегрузки в погружном насосе, что показано в сегменте $b-c$. Когда ток возрастает до установленного значения отключения при перегрузке, двигатель продолжает работать при установленном значении перегрузки в течение некоторого времени. Сегмент $g-1$ указывает на то, что ток внезапно обнуляется. После длительной работы в режиме перегрузки добыча жидкости уменьшается из-за большого выбора нагрузки, как показано в сегменте b - с. После остановки производство жидкости продолжает снижаться, как показано в сегменте $\mathrm{g}-\mathrm{h}$. Сегмент e - f указывает на то, что соотношение газ - жидкость не изменяется в течение всего рабочего режима.

Случай 4: Аналогично, сегмент $\mathrm{a}-\mathrm{b}$, как показано на рис. 2, указывает на то, что УЭЦН работает правильно. Затем давление на входе часто колеблется, поэтому УЭЦН сильно подвержена влиянию газа. В то же время грузоподъемность насоса становится слабее. Добыча газа увеличивается в сегменте b - c. Кривая тока колеблется из-за большего количества свободного газа. Более того, ток нестабилен и регулярно колеблется. Давление на входе и выходе незначительно падает, добыча жидкости немного увеличивается или остается неизменной в сегменте $b-c$. Прежде всего, соотношение газ - жидкость сильно колеблется и увеличивается в сегменте $\mathrm{b}-\mathrm{e}$.

Случай 5: Сегмент $a-b$, как показано на рис. 2, указывает на то, что двигатель работает нормально. Когда газ подвергается 
сильному влиянию УЭЦН, в насос поступает много газа, добыча жидкости имеет тенденцию к снижению в сегменте b - 1, добыча газа имеет тенденцию к увеличению колебаний в сегменте $\mathrm{b}-\mathrm{d}$, a газ - коэффициент ликвидности растет в сегменте $b-c$. Когда уровень рабочей жидкости близок к входу всасывания насоса, ток значительно колеблется, и тенденция к снижению неустойчива. Более того, избыточное газосодержание блокируется в насосе, ток быстро падает в сегменте м - p из-за защиты от недогрузки. В состоянии остановки производственные параметры находятся в нисходящем тренде.

Случай 6: Когда движущаяся поверхность падает, тогда давление масла падает, и УЭЦН работает ненормально. Характеристики параметров очевидны по однофазному току. Существует также значительное колебание в соотношении газ - жидкость. Аналогично, сегмент $a-b$, как показано на рис. 2, указывает на то, что УЭЦН работает должным образом. Ток ниже, чем номинальное значение в сегменте $\mathrm{b}-\mathrm{c}$ и его устойчивый спуск в соответствии с определенным наклоном. Ток быстро падает в сегменте $\mathrm{m}-\mathrm{p}$ из-за отключения защиты от недогрузки. После некоторого времени отключения УЭЦН он снова запускается в сегменте $\mathrm{b}-\mathrm{e}$.

Случай 7: Когда эксплуатационная колонна выходит, жидкость вытекает из трубы под разницей давлений внутри и снаружи трубы. Зависимость между давлением и временем исходной колонны повреждается и нагрузка уменьшается. Аналогично сегмент $\mathrm{a}-\mathrm{b}$, как показано на рис. 2, показывает, что двигатель работает правильно. Производство жидкости ниже номинального значения в сегменте $b-d$. В момент условий работы в сегменте $b-e$. В сегменте $b-$ е резко снижается давление масла. Однако однонаправленный ток не изменяется - это условие в сегменте $\mathrm{a}-\mathrm{c}$.

Случай 8: Когда напряжение погружного двигателя превышает его номинальное значение, УЭЦН не находится в нормальном рабочем диапазоне, а насос грузоподъемности поднимается. Аналогично, сегмент $\mathrm{a}-\mathrm{b}$, как показано на рис. 2, указывает, что УЭЦН работает правильно. Мощность двигателя возрастает в сегменте b - c. Затем в сегментах $b-\mathrm{m}$, сегменте $\mathrm{c}-\mathrm{f}$ и сегменте $\mathrm{b}-\mathrm{h}$ растут единичные потоки, производство жидкости и газа. Когда ток достигает установленного тока перегрузки в сегменте $\mathrm{b}-\mathrm{c}$, после некоторой работы УЭЦН име- ет отключение защиты от перегрузки в сегменте $\mathrm{m}$ - n. Ток, мощность производства жидкости, добыча газа и давление масла падают в сегменте $f-g$ и сегменте $h-1$, сегменте $\mathrm{d}-\mathrm{e}$ и сегменте $\mathrm{o}-\mathrm{p}$ в процессе $\mathrm{n}-\mathrm{o}$ сегмента соответственно. Прежде всего, соотношение газ - жидкость всегда находится в нормальном диапазоне в $\mathrm{a}-\mathrm{p}$ - сегменте в течение всего рабочего режима.

Случай 9: Когда напряжение погружного двигателя ниже его номинального значения, УЭЦН не находится в нормальном рабочем диапазоне, насос грузоподъемности снижается. Аналогично, сегмент $a-b$, как показано на рис. 2, указывает, что УЭЦН работает правильно. Питание двигателя снижается в сегменте $\mathrm{b}-\mathrm{d}$. Затем текущие добывающие мощности и добыча газа снижаются в сегментах $\mathrm{b}-\mathrm{d}, \mathrm{b}-\mathrm{m}$ и $\mathrm{b}-\mathrm{h}$ соответственно. Но ток достигает установленного тока недогрузки в сегменте $\mathrm{d}-\mathrm{e}$. Поработав некоторое время, УЭЦН имеет отключение защиты от перегрузки в сегменте e - f. Ток, производительность жидкости, добыча газа и давление масла падают в сегменте $\mathrm{f}-\mathrm{g}$, сегменте $\mathrm{h}-1$ и сегменте $\mathrm{d}-\mathrm{e}$ в процессе сегмента $\mathrm{f}-\mathrm{g}$ соответственно. Соотношение газ - жидкость всегда находится в нормальном диапазоне в сегменте $\mathrm{a}-\mathrm{p}$ во всем рабочем состоянии.

\section{Выводы}

После дискретной выборки сигнала среднее значение сигнала в нормальном рабочем периоде рассматривается как стандартный сигнал. Нормализуя сигнал и сравнивая данные, полученные при каждой выборке, различается градусное отклонение текущего одноточечного рабочего состояния и нормальное состояние.

В статье предложена схема мониторинга рабочего состояния электроцентробежных насосов на основе электрических и эксплуатационных параметров устья скважины. При этом статус подземной добычи отличался различными параметрами ручного ввода в разных скважинах, что улучшило эффективность распознавания при диагностике неисправностей. В соответствии с фактическими случаями составлены графики параметров девяти типов электроцентробежных насосов. Были точно определены причины неисправностей с помощью анализа принципиальных схем. Алгоритмы, разработанные с функцией извлечения, своевременно устраняли неисправности моделей. Кроме того, было проведено больше экспериментов в электроцентробежных насосах, 
а результаты испытаний показали, что алгоритмы достигли требований с помощью практических инженерных применений.

\section{Список литературы / References}

1. Большунов А.В. Современные методы диагностики фактического состояния установок электроцентробежных насосов // Успехи современного естествознания. 2019. № 12-2. C. 249-253.

Bolshunov A.V. Modern methods for diagnosing the actual state of electric centrifugal pump installations // Advances in current natural sciences. 2019. № 12-2. P. 249-253 (in Russian).

2. Габдрахимов М.С., Фахриева К.Р. Динамические нагрузки скважинного оборудования и виброзащита УЭЦН / Оборудование и технологии для нефтегазового комплекса. 2013. № 5. C. 17-21.
Gabdrakhimov M.S., Fakhrieva K.R. Dynamic loads of downhole equipment and vibration protection of the ESP // Equipment and technologies for the oil and gas complex. 2013. № 5. P. 17-21.

3. Maystrenko A.V., Svetlakov A.A., Gandzha T.V., Dmitriev V.M., Aksenova N.V. Application of numerical signal differentiation methods to determine stationarity of a process. Petroleum and Coal. 2017. V. 59. I. 3. P. 311-318.

4. Гнеденко Б.В., Беляев Ю.К., Соловьев А.Д. Математические методы в теориинадежности. М.: Наука, 1965. $524 \mathrm{c}$.

Gnedenko B.V., Belyaev Yu.K., Soloviev A.D. Mathematical Methods in Theory reliability. M.: Nauka, 1965. 524 p.

5. Dmitriev V.V., Gandzha T.V., Dolganov I.M., Aksenova N.V. An algorithm to improve the speed and accuracy of analysis of chemical process systems operation. Petroleum and Coal. 2017. V. 59. I. 3. P. 429-441. 